semble justiciable devant le « tribunal de l'histoire ", si tribunal il y a. Ce sont les mille et un tours de cette alchimie subtile, indispensable aux sociétés ainsi qu'aux individus qui les composent, que les historiens devront comprendre et analyser, sans commettre - chose difficile - trop de contresens sur ces tours de magie dont les causes ou les ingrédients nous échappent trop souvent.

$\mathrm{Si}$ l'image de par sa nature représente un relais essentiel dans la constitution des grands mythes de l'histoire de France et dans la "mémoire " patriotique, ce pourquoi les historiens se penchent avec attention sur les illustrations des livres scolaires, analysent l'évolution de la représentation de scènes ou de personnages clés de cette mythologie, il ne faut pas mesurer l'importance d'un mythe à l'importance de la production iconographique qu'il aura suscitée. Au cours de ce colloque a été mise en lumière la faiblesse de la filmographie touchant toutes les périodes de la Révolution française; à l'inverse, on a pu constater qu'une image "manquante" comme celle de la peinture antique disparue a pu jouer un rôle considérable au XVIII ${ }^{e}$ siècle dans les débats entre Antiques et Modernes, obligeant les Modernes à se définir par rapport à un modèle d'autant plus parfait qu'il n'était connu que par les textes et qu'il avait été reconstitué, fantasmé, halluciné.

Il n'est pas question de retranscrire dans un compte rendu succinct la complexité des méthodes sur lesquelles les auteurs s'étendent plus ou moins longuement, le lecteur aura trouvé dans ces quelques lignes, je l'espère, l'envie de recourir aux textes et de se plonger dans ces communications où il trouvera amplement matière à réflexion. Nous conclurons avec J. R. Louvet : " Symptômes d'une facette du développement de l'historiographie actuelle, les travaux sur les images ouvrent de nouveaux champs d'études sans encore être novateurs. Ils marquent un commencement dont ces textes donnent un indice, une sorte d'acte de naissance multiplié. »

Mireille GueIssaz.

\title{
EXPRESSIONS DU RELIGIEUX
}

Hervé Martin, Le Métier de prédicateur à la fin du Moyen Âge : 1350-1520. Paris, Cerf, 1988. 14,5 × 24, 720 p. ("Histoire").

En ces temps d'explosion planétaire des moyens de communication, il n'est pas étonnant de voir l'historien décrire le milieu des gens de la parole, décrypter leur 
message et évaluer son impact. Ce qui est plus extraordinaire, c'est le choix de cette période, longtemps mal aimée, volontiers ressentie comme décadente, qui nous semble aujourd'hui si proche dans son malaise et ses quêtes. Cet ouvrage est la version réduite d'une thèse de doctorat ès lettres exemplaire, dont le défi consistait à décrire sur deux siècles l'activité de 1947 prédicateurs attestés et de 849 locuteurs probables dans la France septentrionale.

Cette activité a très vite été accaparée par les mendiants, spécialement les Franciscains, les premiers à avoir compris les règles de la communication en milieu urbain. Avec leurs grands rivaux dominicains, ils arrivent à un quasimonopole de la prédication au $x v^{e}$ siècle. Ils exercent alors un véritable métier, qui requiert une longue préparation intellectuelle et pratique, et la soumission à une discipline sociale, aux règles proches de celles des corporations. Pour la première fois, nous possédons une sociologie de ces professionnels de la parole, plus ou moins célèbres, qui quadrillent le pays et savent imposer leur savoir-faire à des foules urbaines avides de rhétorique et de théâtre. Un public exigeant, de plus en plus qualifié, sachant juger à la fois la science et l'éloquence, volontiers nombriliste, désireux d'entendre parler de lui-même et de sa conversion, fût-ce au prix de quelques vérités désagréables.

L'étude classique de sociologie est complétée par une époustouflante et plus difficile analyse du discours, à travers $\mathbf{7 7 0}$ sermons, dont la moitié en latin et les trois quarts manuscrits. La lexicologie, la sémantique structurale et l'analyse de contenu, celle de l'appareil formel de l'énonciation enfin, conduisent à quantité de conclusions nouvelles et surprenantes sur le paysage mental de la fin du Moyen Âge. Dans cette construction scolaire qu'est le commun des sermons, la Bible sourd de partout; elle est une référence à la fois littérale et morale tout à fait fondamentale, n'en déplaise aux détracteurs des siècles suivants. Mais il est vrai qu'il s'agit d'une Bible en miettes, allégorisée, réinterprétée et glosée, alors que l'Humanisme redécouvre justement l'émotion vigoureuse du recours aux textes primitifs. Le sens simple et immédiat est trop souvent court-circuité, pour la plus grande joie des masses avides de bons mots, mais à la déception grandissante des élites qui commencent à vouloir aborder le texte lui-même.

Ces sermons, passablement rébarbatifs pour nous, s'inscrivent dans des structures mentales qui les rendent parfaitement efficaces dans la culture urbaine de leur temps. Ils tendent parfois à la cuistrerie, à la langue de bois même; ils martèlent et rabâchent une foi uniforme et une morale cohérente, qui sont des éléments de l'unanimité communautaire. Ils transmettent un catéchisme élémentaire, ils mettent à la mode des pratiques de dévotion; ils diffusent surtout une morale rigoriste, car il faut bien éduquer à la confession annuelle, dont la mise en place est la grande aventure de l'Église de ce temps.

La dérive métaphorique était alors fatale. Elle était rendue nécessaire par la tâche assignée aux prédicateurs de décrire une pensée théologique sous les traits d'une pensée concrète. On peut regretter l'absence d'une étude de l'adaptation des sermons aux courants théologiques dominants, qui conditionnent malgré tout l'espace du croyable. Mais il est vrai que même une thèse d'ancien régime ne peut tout voir, sous peine de devenir un monstre. L'étude technique permet cependant d'observer l'étrange travail alchimique de la métaphore, qui rend possible tout un 
jeu de correspondances entre naturel et surnaturel, corporel et mental, intemporel et historique.

Cette dérive n'a nullement empêché la poursuite du succès des exempla rassemblés par les générations précédentes. Mais ceux-ci ne se renouvellent plus à la fin du Moyen Âge. Leurs sortilèges servent toujours à faire rire, faire voir, faire rêver, faire dire ; bref, à rendre le discours efficace à l'aide de recettes éprouvees. Il est bien difficile de savoir jusqu'à quel point va leur réussite, car nous ne saurons jamais sonder les reins et les cœurs. Mais ceux-ci témoignent au moins de la réalité et du succès médiatique d'une pastorale de masse.

Les prédicateurs ont, en effet, su s'adapter aux temps et aux lieux, aux publics et à leur langue, au moins en ville. Une certaine lassitude se fait cependant jour au début du $\mathrm{XVI}^{\mathrm{e}}$ siècle; trop longtemps abreuvés d'une rhétorique aux effets trop attendus, quelques croyants sont en quête d'émotions nouvelles. Les prédicateurs se trouvent contraints de chercher d'autres bases d'expression. L'aventure de la Réforme protestante et celle de la Contre-Réforme sont inscrites dans ce défi de la transmission du christianisme dans de nouveaux mots.

Nicole LEmAîtRe.

Nicole LEMAîtRE, Le Rouergue flamboyant. Le clergé et les fidèles du diocèse de Rodez, 1417-1563. Préf. Jean Delumeau. Paris, Cerf, 1988. $15 \times 24,658$ p. ("Histoire").

Nicole Lemaitre apporte, dans Le Rouergue flamboyant, une contribution majeure à l'histoire religieuse de la France, justement récompensée par le prix du "jeune historien" que lui a décerné Notre Histoire et la $1^{\text {re }}$ médaille des Antiquités (Académie des inscriptions et belles-lettres). Elle a, en effet, construit une étude dense, très informée, sur la vie religieuse du clergé et des laïcs dans l'attachant Rouergue aux $\mathrm{Xv}^{e}$ et $\mathrm{XV}^{e}$ siècles; elle en restitue les aspects déterminants avec une sympathie chaleureuse, à partir d'une scrupuleuse analyse des sources; la problématique innovante qui sous-tend cette thèse se révèle, en effet, par esprit de rigueur et modestie, sous des apparences de classicisme.

Ainsi le plan s'ouvre-t-il, dans la meilleure tradition de l'école historique française, par un tableau géographique des diocèses de Rodez et de Vabres, et s'articule en quatre séquences chronologiques destinées à faire ressortir l'évolution de la société cléricale et des comportements religieux entre la fin de la guerre de Cent Ans et le début des guerres de religion. $N$. Lemaitre brise délibérément les cadres chronologiques traditionnels et inadéquats pour s'interroger sur la définition et l'apparition des phénomènes qui permettraient de distinguer l'Église et la religion des "Temps Modernes " de celles du " Moyen Âge ".

Une première partie expose les "Cadres hérités". Les six évêques qui se succèdent sur le siège de Rodez, de Vital de Mauléon (1417-1429) au cardinal Georges d'Armagnac (1530-1560), exercent leur ministère dans les structures 
ecclésiastiques définies aux XIII $^{e}$ et XIV $^{e}$ siècles. L'évêque de Rodez était un seigneur riche, exerçant l'autorité sur la cité de Rodez puis, après 1530, les pouvoirs comtaux. Il contrôlait avec une cour épiscopale importante, mais avec peu d'officiers diocésains intermédiaires, un clergé dont la situation bénéficiale était caractéristique de la fin du Moyen Âge (dissociation fréquente entre le titulaire et le desservant de la cure). L'autorité spirituelle de l'évêque n'a cessé, au $X I V$ siècle, d'imposer la perspective de la reformatio in membris du clergé définie par les statuts de 1289 ; les prélats ont veillé à la régularité des services pastoraux et lutté contre l'absentéisme.

$A u X v^{*}$ siècle, un effort de réforme tenace relève le diocèse ravagé par la guerre, longtemps divisé par le Grand Schisme, et réalise la "construction d'une identité religieuse rouergate ". L'instrument essentiel de la réforme est la visite pastorale. Sa répétition, son ampleur croissante, la méticulosité accrue des Ordonnances de Georges de La Tour puis de Bertrand de Chalençon, ont assuré un redressement matériel significatif des lieux de culte, notamment des annexes. Un mobilier liturgique restauré, des livres en suffisance, des revenus bénéficiaux améliorés assurent un cadre plus décent pour le culte que les évêques s'efforcent de plus en plus de séparer des occupations et comportements profanes. Les visiteurs s'intéressent aux mœurs et à la religion des paroissiens; en revanche, la réforme du clergé passe par un contrôle plus systématique des capacités à l'entrée en cléricature et lors de l'accession aux ordres. Cette réforme rencontre des limites dans la non-résidence des curés, dans le concubinage qui paraît toucher $20 \%$ des prêtres, même si la communauté villageoise, de connivence avec ses pasteurs, étouffe quelquefois le scandale. Dans l'ensemble, c'est un clergé en net progrès de connaissance, de dignité et de zèle pastoral que rencontre, au début du $\mathrm{XvI}^{\mathrm{e}}$ siècle, le nouvel évêque François d'Estaing.

N. Lemaitre consacre un grand tiers de son ètude à l'épiscopat de ce prélat (1504-1530). Elle dresse un bilan de la vie religieuse et de l'état du clergé au début des «temps modernes". C'est le temps de "l'Enracinement», de l'identité renforcée du clergé recruté en majorité dans son propre diocèse, qui s'incarne dans l'image du prélat, grand seigneur local, doux, affable, dont les visites confirment la capacité de proximité avec les fidèles. Mais $\mathbf{N}$. Lemaitre donne de François d'Estaing un portrait débarrassé de ses "douceurs hagiographiques" post-tridentines, restitue la figure plus forte et contrastée d'un évêque sincèrement réformateur mais sourcilleux sur les prérogatives et droits de sa fonction, désireux d'accroître la conscience du sacré dans une liturgie plus belle. Mais il est incapable de concevoir un remède efficace contre le cumul et l'absentéisme. L'augmentation des revenus des bénéfices permet en effet d'assurer la subsistance d'un prêtre suppléant le curé et contrôlé dans ses capacités au moment de l'ordination. Cependant, la multiplication des communautés de prêtres-filleuls nés dans la paroisse limite l'effort de contrôle, car elles s'organisent sans licence épiscopale.

Au cœur de la société laïque rurale, comme au sein de ces confréries de prêtres qui en sont l'expression la plus proche dans le clergé, se discernent les premières inflexions de comportement qui annoncent une dévotion plus dépouillée et personnelle; ils sont des signes ténus dans une structure religieuse homogène 
mise en place au cours des siècles précédents. Le culte des reliques, le rituel des morts organisés autour de l'accumulation des suffrages des vivants, les confréries religieuses ordonnent une piété communautaire et sociale que la mort n'interrompt pas. Ce qui change est une conscience plus forte du sacré induisant des attitudes formelles de respect et de respectabilité. Communautés de prêtres et confréries de laïcs abandonnent après 1530 la convivialité du banquet. Le culte marial reste discret, celui de l'Eucharistie connaît une intensité nouvelle. Les legs testamentaires expriment une nouvelle approche de la pauvreté : au pauvre de Dieu, intercesseur idéal, succède le pauvre vertueux, dont la dignité est définie par le testateur.

A peine épanouie, cette identité est menacee par l'apparition de la Réforme protestante que le fastueux cardinal d'Armagnac, protégé de Marguerite de Navarre, figure du prélat romain et humaniste, trop peu résident dans son diocèse, est incapable de prévenir. Une petite minorité de prêtres, fortement protégés, lancee dans une course effrénée aux bénéfices, est seule passée au travers des prescriptions canoniques en général mieux respectées que sous François d'Estaing : voici une nouvelle démonstration que la diffusion du protestantisme ne peut s'expliquer seulement par la protestation contre les abus du clergé devenu indigne. Cependant, l'idéal gersonien du prêtre, publié avec les statuts (1552), est devenu anachronique; le contrôle insuffisant des collations ne peut compenser la surveillance des libraires qui s'installent en Rouergue et des livres d'inspiration luthérienne qui y circulent. En 1554, des communautés protestantes sont organisées à Villefranche, Millau, Sauveterre et dans les campagnes des marges sud et ouest du diocèse. Le ralliement des autorités consulaires de Millau explique ici le succès durable du calvinisme, diffusé parmi les artisans et les marchands, tandis que la brutalité des seigneurs huguenots de Villefranche détermine une réaction catholique encore plus sanglante ; Rodez, ville d'hommes de loi et de clercs, s'est au contraire définitivement fermée aux protestants. Les "bastions" sont fixés avant que n'èclatent les Guerres de religion.

La variété des problèmes abordés par $\mathrm{N}$. Lemaitre, le volume d'informations fourni par un abondant dépouillement de sources sont complétés par un dossier de 20 planches photographiques, 59 cartes, 9 graphiques et 76 tableaux statistiques; on regrettera que la rapidité de publication de la thèse n'ait pas permis à l'auteur de réviser la mise en page des tableaux, dont la lecture manque parfois de clarté, et qui se trouvent souvent en discordance avec les renvois du texte. Quelle que soit l'ampleur du sujet, il imposait des choix ; certaines affirmations suscitent, par conséquent, chez le lecteur quelques réactions (dans une perspective "médiéviste") ou des souhaits d'éclairages complémentaires. Ainsi, le rôle attribué à la guerre de Cent Ans pour expliquer l'absence en Rouergue de prêtres originaires de Guyenne et de Poitou (p. 170) laisse quelque peu perplexe, alors que des clercs sont venus de Gascogne, pourtant également sous domination « anglaise ». Le formulaire du testament de Guillaume Barès (p. 338) évoquant au milieu du $\mathrm{xv}^{e}$ siècle le jugement personnel à l'heure incertaine de la mort paraît s'accorder avec la spiritualité des Artes Moriendi; peut-être, sous bénéfice de connaître sa teneur intégrale, n'est-il précoce que par la référence à saint Paul et saint Mathieu... Le "geste médiéval ", évoqué p. 341, consistant à être mis sur le 
" brancard des pauvres", est sans doute une coutume rouergate? Nous n'avons jamais rencontré cette pratique, par exemple, dans de nombreux testaments italiens contemporains.

Deux sources évoquées par l'auteur auraient mérité sans doute une étude plus détaillée. A propos de l'enquête de 1504 sur l'élection épiscopale de François d'Estaing, premier document hagiographique sur le prélat (p. 265-266) N. Lemaitre n'a pas confronté les stéréotypes qu'elle a relevés à d'autres " images » de saints évêques qui auraient montré la tradition à laquelle les clercs du début $d u x{ }^{e}$ siècle cherchaient à se rattacher. De même, un recueil de sermons est simplement mentionné (p. 330) sans que les thèmes principaux qu'il contient soient seulement évoqués. L'étude approfondie de ces textes destinés à l'édification des fidèles révélerait peut-être des indices sur la transmission des idées, aux lisières de l'orthodoxie religieuse dans la période comprise entre 1467 (dernière manifestation " hérétique " liee au Schisme) et 1530 (apparition de la Réforme), au cours de laquelle le fil des convictions religieuses en marge de l'organisation ecclésiale se perd.

D'autres contributions de $\mathbf{N}$. Lemaître répondront sans doute à ces interrogations, complétant la perception convaincante que l'auteur donne de la chrétienté rouergate. Elle y est parvenue grâce à une parfaite maîtrise des techniques d'investigation les plus modernes. Elle a su éviter la fascination qu'elles engendrent parfois chez l'historien : ainsi l'informatique, qui permet de reconnaître la composition et l'analyse factorielle des revenus bénéficiaux; ainsi l'anthropologie, dans le domaine où se déploie, avec plus d'aisance, son analyse des attitudes religieuses. En moderniste qui connaît la valeur des données sérielles et sait combien peuvent être fragiles les conclusions bâties sur des faits isolés et des documents épars, elle a su éviter le primat du singulier mental ou anthropologique. En témoignent la grande prudence méthodologique dont elle fait preuve face aux formulaires des testaments, et toutes les fortes pages qui montrent que la cohésion socio-religieuse entre les prêtres et les fidèles était un "rempart ", plus efficace contre les idées de la Réforme que le degré de formation religieuse des clercs. Telle, également, l'étude qui souligne la densité des liens entre les familles et leurs morts dans la ruralité montagnarde du Rouergue. Jusqu'à la fin du $x v^{e}$ siècle, le choix de sépulture était guidé par une logique lignagère (la tombe des parents plutôt que la paroisse), l'enterrement dans l'église exceptionnel. $\mathrm{N}$. Lemaître verse ainsi une pièce importante au dossier de preuves (on en trouverait de nombreuses, semblables, en Italie) qui relativisent l'idée de l'effondrement des structures sociales, le déracinement des populations et la montée de l'individualisme à la fin du Moyen Âge. Les observations contraires de J. Chiffoleau en Comtat Venaissin en acquièrent d'autant plus le relief de la singularité. Ainsi le protestantisme n'est-il pas d'abord une réponse à un ébranlement existentiel. 
Léo Steinberg, La Sexualité du Christ dans l'art de la Renaissance et son refoulement moderne. Trad. de l'anglais Jean-Louis HouDEBINE, préf. André Chastel. Paris, Gallimard, 1987. $19 \times 24,265$ p., ill., bibliogr., index ("L'infini »).

Publié à New York chez Pantheon Books, en 1983, sous le titre The Sexuality of Christ in Renaissance of Art and in Modern Oblivion, la traduction française de cet ouvrage de Léo Steinberg est d'un abord contrarié par un titre racoleur, qui masque plus qu'il n'annonce le propos ponctuel mais très profondément résonnant de son auteur.

Propos précisément formulé : l'exposition délibérée des genitalia du Christ dans les images de la Renaissance (la démonstration de l'auteur puise ses exemples dans la peinture du Trecento et s'étend jusqu'aux images du XvII ${ }^{\mathrm{e}}$ siècle) est l'expression d'une dévotion qui insiste sur l'humanité du Christ (Steinberg retie ${ }_{\text {. }}$ le terme anglais d'humanation, supplanté au XvIr' siècle par celui d'incarnation), conformément aux développements d'une théologie que de très nombreux textes ou sermons établissent, et que le christianisme de la Renaissance fait prévaloir. Humanation, c'est-à-dire prise en charge de la condition humaine avec toutes ses caractéristiques et ses exigences physiques : nécessité de se nourrir au sein maternel de la petite enfance puis à Emmaüs, preuve vivante que fut assumée, de la naissance à la Résurrection, la chair humaine; capacité de procréer, sans laquelle l'humanité du Christ resterait virtuelle, alors que le catalogue des images invoquées par Steinberg insiste sur l'identité et la puissance sexuelles du Christ, sans contradiction avec le fait que cette puissance soit maîtrisée et demeure en suspens durant tout son ministère.

La cohérence d'une sensibilité chrétienne, dont les interprétations rejoignent par ailleurs le symbolisme des anciens mystères païens, nuine ici l'explication naturaliste et anecdotique de thèmes picturaux : nativité, Sainte Famille, circoncision, crucifixion, trône de grâce - où le sexe est offert au regard, au couteau, à l'attouchement, images qui ne sauraient relever de la scène de genre ni se justifier par une esthétique du nu ou une exaltation du modèle antique propre à la Renaissance. En recourant aux écrits des Pères de l'Église, passés et contemporains, l'auteur montre au contraire comment l'humanation ainsi détaillée réconcilie les deux dimensions, humaine et spirituelle, du Christ, restaure " la possibilité d'une nature humaine sans culpabilité humaine" (p. 39), "l'innocence primitive perdue avec Adam " (p. 42).

Circoncision et crucifixion sont les moments extrêmes, symboliquement les plus chargés, de cette rédemption. Sceau de l'ancienne alliance entre Dieu et ses élus, que le baptême, sacrement de la nouvelle alliance, circoncision spirituelle, invalidera (voir La Circoncision de Mantegna, reproduite p. 73), la circoncision, en confirmant l'incarnation, apparait comme un premier acompte versé au profit de l'humanité rachetee par le sang du Christ, une préfiguration de la Passion. Car le sang versé sous le couteau du prêtre annonce le sang jailli du cœur transpercé sur la croix, et le cycle ainsi accompli, déroulant simultanément une vie d'homme et une construction hautement symbolique, justifie dans maints tableaux le filet 
sanglant qui reflue du sein à l'aîne du crucifié, lieu du sacrifice (voir notamment La Pieta de Jean Malouel ou Le Retable de Saint-Denis d'Henri Bellechose, reproduits p. 79 ).

La symbolique chrétienne subtilement articulée que mettent en pratique les peintres de la Renaissánce fut, selon Steinberg, refoulée par les siècles suivants, qui ont écarté ou ignoré des notions théologiques complexes devenues, progressivement, inopérantes. L'intérêt de sa démonstration tient à la leçon de lecture des œuvres qu'il propose, à la détermination de critères exacts d'interprétation aptes à en rendre compte, à la mise en question des catégories d'école (naturalisme, réalisme) qui évident le contenu des peintures. Rendre tangible, dans ces représentations, la double identité du Christ, Dieu et homme, c'est aussi, métaphoriquement, restaurer toute la portée de l'image, forme et sens se choisissant mutuellement. De la justesse, du naturel de leur rencontre, de leur coïncidence, naîtra l'efficacité de la représentation, que l'on nomme parfois la beauté.

Anne ReINBOLD.

François LAPLANCHE, L'Écriture, le sacré et l'histoire. Érudits et politiques protestants devant la Bible en France au XVIF siècle. Amsterdam/Maarsen, A.P.A. Holland University Press (Diff. Presses universitaires de Lille), 1986. $15,5 \times 23$, XXXVI-1 020 p.

Comment s'opèrent les changements dans l'histoire ? Qu'est-ce qu'une « transformation de mentalité $"{ }^{9}$ ? Telles pourraient être les questions que pose et aide à résoudre la monumentale thèse de François Laplanche. Pour étudier sur la longue durée, du XVI ${ }^{e}$ au XVIII ${ }^{e}$ siècle, les évolutions qui ont mené de l'humanisme aux Lumières, F. Laplanche s'est penché sur un milieu religieux et social : les professeurs de l'Académie protestante de Saumur au XvIr siècle et les pasteurs parisiens qui leur étaient liés. Ce groupe, en effet, lui a paru cristalliser des questions centrales que les humanistes avaient, un siècle plus tôt, dégagées, et qui portaient sur le texte, sur la Bible. Ce n'est pas pour étonner de la part de protestants, mais cela place Saumur dans le prolongement de ceux qui s'interrogeaient sur les corruptions du texte, qui se livraient, depuis Scaliger, à la « critique " textuelle, et essayaient de retrouver l'origine, lieu de la vérité, en deçà des inévitables altérations du temps.

Saumur recevait aussi l'héritage des “politiques ${ }^{10}$, qui, depuis Michel de

9. P. 378.

10. P. 101 et sq. 
l'Hospital "' et les gallicans ${ }^{12}$, ne voyaient d'autre moyen de restaurer la paix du royaume que dans une séparation du temporel et du spirituel, et dans l'élaboration d'une éthique politique reposant sur des bases " naturelles», sur la " conscience", la nature humaine, la patrie ${ }^{13}$.

A vrai dire, les deux préoccupations étaient, malgré les apparences, étroitement liées, et c'est ce qui confère une rigoureuse unité à la thèse de F. Laplanche qui, sans perdre de vue son objectif, peut passer des questions textuelles aux questions politiques et vice versa. Au centre de cette histoire, deux hommes, Louis Cappel et Moyse Amyraut, qui, chacun à sa façon, ont permis le passage d'une mentalité à l'autre. Cappel, auteur d'une Critica sacra au titre significatif, montra que le texte lui-même était soumis à l'histoire, que l'Antiquité n'était pas toujours signe de vérité, que la vérité s’atteignait par un travail, un exercice de la raison. Il n'y aurait pas selon lui transmission passive depuis l'origine, il fallait établir les faits, la vérité étant elle-même l'origine cherchée, non donnée mais produite ${ }^{14}$.

Amyraut veut unir la théologie et le droit ${ }^{15}$, et sa visée est éthique; lui aussi s'appuie sur la nature, sur la raison, et il peut poser que " omne verum a Deo " ${ }^{16}$, tendant à élaborer une politique où temporel et spirituel soient séparés ${ }^{17}$; d'où son projet d'une "morale chrétienne ${ }^{18}$. Ce projet n'était pas sans conséquences : du point de vue théologique, c'était s'opposer à une conception « nominaliste " (mais aussi d'un calvinisme un peu simpliste) d'un Dieu capricieux dont la volonté constituerait le bien ${ }^{19}$, et à une conception extrinséciste de la grâce. C'était aussi prendre ses distances vis-à-vis de l'Ancien Testament en se tournant plutôt vers les leçons du Nouveau ${ }^{20}$.

Pour les hommes de Saumur, comme pour les humanistes, s'esquisse une anthropologie qui permet de repenser les conceptions de l'inspiration scripturaire et de la prophétie, et de dévaloriser l'apocalyptique : en distinguant, comme Bèze, Drusius, Caméron, Grotius (chacun de façon différente), des niveaux d'inspiration, des types de textes inspirés, ou des degrés de prophétie, les hommes de Saumur pouvaient promouvoir une interprétation "morale " et "utile " de la Bible, et, en dévalorisant les phénomènes extatiques ou prophétiques, s'opposer aux modernes Enthousiastes dont les audaces risquaient d'entraîner dans les aventures politiques le protestantisme menacé. Ici encore les critères de la raison et de la morale organisaient à la fois l'interprétation du texte et la pensée politique. Ce qui se met donc en place, c'est une méthode de critique du texte consciente de ses présupposés, donc une pratique textuelle et les fondements

11. P. 122 et sq.

12. P. 134 et sq.

13. Cf. p. $116-117$.

14. Cf. p. 186 et sq., 193, 223-224, 234, 310, 372-373 et passim.

15. P. 379 et sq.

16. P. 388, 403, 404 .

17. P. 520-521.

18. P. 475 et sq

19. P. 385 .

20. P. 405 et sq. 
d'une pratique politique. Moins que par le détail des thèses théologiques ou des interprétations de passages de la Bible, c'est par l'élaboration de cette double pratique, où s'imposent des critères "modernes", que l'influence de Saumur se révélera durable, et $\mathrm{F}$. Laplanche pourra consacrer la dernière partie de son enquête à la double postérité de Saumur, à la façon dont se répandirent et prirent force d'évidence les idées et les critères de Saumur, soutenus par d'irréversibles pratiques.

Mais, en même temps, il pourra montrer comment la raison, la nature, l'utilité devinrent critères autonomes en se détachant de leurs origines religieuses. Une des conséquences, c'est que la mystique, le piétisme et l'illuminisme s'opposeront désormais à une religion de plus en plus morale, où enthousiasme, extase et prophétie n'apparaîtront que comme survivances, superstitions, ou comme ce qui, à la manière du miracle, vient autoriser de l'extérieur, et confirmer pour l'incroyant, un corpus de vérités (au pluriel) et des leçons à l'évidente utilité morale et sociale.

Le millier de pages à la typographie serrée qu'a consacré F. Laplanche à cette évolution ne peut être résumé, sous peine de trahir le foisonnement des faits et des interprétations, la finesse nuancee des lectures et des conclusions. En tout cas, il a admirablement décrit et expliqué une des voies de la grande mutation occidentale dont, bien loin d'être un siècle d'accomplissement, le XVII ${ }^{e}$ siècle est l'opérateur. Il y a d'autres voies que $F$. Laplanche lui-même signale : Henning Graf Reventlow a, dans un livre aussi monumental ${ }^{21}$, étudié les rapports de la pensée anglaise avec la Bible, de l'humanisme aux Lumières, et ce qui nous frappe c'est que le livre de H.G. Reventlow comme celui de F. Laplanche montrent très bien ce que ce dernier affirmait avec pertinence, dans un article des Annales ${ }^{22}$, qu'un système orthodoxe ne chancelle pas sous la poussée de forces extérieures, mais qu'il s'effrite de l'intérieur. Cette conclusion peut être tirée de l'étude de bien d'autres filiations : il n'est pas impossible de tracer une ligne continue de la mystique du $\mathrm{XVII}^{e}$ siècle et de Fénelon, du théocentrisme de Malebranche, ou de la theologie du P. Petau, jusqu'à la religion des Lumières : à chaque fois, c'est de l'intérieur d'une confession religieuse et du point extrême qui était visé dans cette confession que, par une sorte de nécessaire logique, est sorti ce qui en devait ètre la négation. Le fait mérite réflexion; outre qu'il dévalorise la naĩve thèse du " complot", il révèle le caractère essentiellement instable, lié au temps et à la société, de toute synthèse et de tout système religieux. C'est ce que l'exemplaire travail de F. Laplanche nous aide à penser. Resterait, après lui et aussi après celui de H.G. Reventlow, à décrire tel ou tel autre "phylum ", qui, lui aussi lié à un temps et à un milieu, aiderait à comprendre le problème précis de la mutation de la pensée et des pratiques du texte et du politique. N'en citons que deux : l'évolution de la théologie catholique, depuis les jésuites des $\mathrm{XVI}^{e}$ et $\mathrm{XVII}^{\mathrm{e}}$ siècles

21. Bibelautorität und Geist der Moderne. Die Bedeutung des Bibelverständnisses für die geistesgeschichtliche und politische Entwicklung in England von der Reformation bis zur Aufklärung, Göttingen, Vandenhoeck \& Ruprecht, 1980.

22. F. LAPLANCHE, "Tradition et modernité au XVII siècle. L'exégèse biblique des protestants français ", Annales ESC, 3, mai-juin 1985, p. 463-488. 
jusqu'à Holden, Petau et Richard Simon et jusqu'au xvIII ${ }^{\mathrm{e}}$ siècle; et surtout, un peu trop négligé par F. Laplanche (mais, écrivait Bremond, "non omnia possumus omnes »!), le spinozisme ${ }^{23}$ : la réédition de La Philosophie interprète de l'Écriture sainte $^{24}$ de Louis Meyer, et des travaux récents sur Bayle ${ }^{25}$ et sur Le $\mathrm{Clerc}^{26}$, pourraient être l'occasion de reprendre cette question du spinozisme et de se demander si des problèmes proprement philosophiques ne sont pas aussi en cause dans la mutation des pratiques et des mentalités que le grand livre de F. Laplanche a si bien analysée.

Jacques Le BRUN.

Historische Kritik und biblischer Kanon in der deutschen Aufklärung. Herausgegeben von Henning Graf Reventlow, Walter SPARN, John WoodBRIDGE. Wiesbaden, Harrassowitz, 1988. $15 \times 23$, VIl-291 p. (« Wolfenbütteler Forschungen », Bd 41).

Ce volume édite les actes d'un colloque tenu du 10 au 15 décembre 1985 à la prestigieuse Herzog August Bibliothek. Comme beaucoup d'ouvrages de cette espèce, il frappe, en première lecture, par la richesse de son érudition et par une inévitable dispersion des thèmes, puisque la plupart des communications sont consacrées à un seul personnage (sauf la deuxième de H.G. Reventlow : "Wurzeln der modernen Bibelkritik» et celle de D. Bourel : « Die deutsche Orientalistik im 18. Jahrhundert "). Cependant, une lecture plus attentive permet de repérer les grands axes selon lesquels s'opèrent les transformations de l'exégèse biblique au temps des Lumières allemandes.

1. L'axe temporel

Toute histoire de la culture gagne à être immergée dans la longue durée. L'éditeur du volume, H.G. Reventlow, dans ses deux contributions (celle citée cidessus et celle intitulée : « Bibelexegese als Aufklärung. Die Bibel im Denken des Johannes Clericus (1657-1736) »), montre à juste titre comment l'exégèse des Lumières s'enracine dans la philologie humaniste $d u x v I^{e}$ siècle. A ce titre, le personnage de Le Clerc, admirateur de Grotius et éditeur d'Érasme, constitue une figure exemplaire. L'impact de l'humanisme sur l'exégèse se marque avant tout

23. P. 518,588 et sq.

24. Louis MEYER, La Philosophie interprète de l'Écriture sainte, trad. du latin, notes et prés. par Jacqueline Lagrée et Pierre-François Moreau, Paris, Intertextes, 1988.

25. Pierre Bayle, Écrits sur Spinoza, textes choisis et présentés par Françoise CharlesDaubert et Pierre-François Moreau, Paris, Berg Intemational, 1983.

26. Maria Cristina PITASSI, Entre croire et savoir. Le problème de la méthode critique chez Jean Le Clerc, Leyde, E.J. Brill, 1987. 
par le privilège accordé au sens littéral. Cette attention, pour se revêtir d'un vocable traditionnel, implique en réalité une déclaration de guerre à toute exégèse reflétant la préoccupation confessionnelle et, quoique adversaires, Le Clerc et Richard Simon témoignent ici d'une commune sensibilité. Des années 1670 à 1720 , cet humanisme va prendre une couleur plus nettement philosophique, en se grossissant de courants qu'on appellera « rationalistes ", pour faire court. Le droit naturel s'impose comme fondement de la paix intérieure et extérieure des États, refoulant ainsi les prétentions des " politiques chrétiennes ". Les exégèses contradictoires des confessions chrétiennes, relatives aux mêmes passages de la Bible, font progresser la cote de la religion naturelle, seule capable de réunir les esprits. Enfin, s'accentue l'allergie aux aspects suprarationnels de la Révélation chrétienne; Dieu et la liberté, la captivité du péché et l'autonomie de l'homme, la simplicité de l'essence divine et la Trinité des personnes, tout cela apparait contradictoire ou au moins inutilement compliqué, par rapport à la pure simplicité de l'Écriture (voir les communications de M. Schloemann sur Baumgarten et de W.E. Müller sur Jérusalem). Ce qui, dans la Bible, ne relève pas de ce christianisme raisonnable doit être imputé aux idées du milieu où ont vécu les auteurs, donc à l'histoire (ceci est surtout vrai pour l'Ancien Testament, mais est appliqué au Nouveau par Reimarus : voir la communication de W. SchmidtBiggemann).

Il devient alors possible et même nécessaire d'étudier la Bible comme n'importe quel autre livre ancien, avec toutes les ressources de la "critique », ce que disent à l'envi J. A. Turrettini, J.J. Wettstein, J. D. Michaëlis, J.S. Semler. En dépit de cette position radicalement désacralisante, les Lumières allemandes gardent un religieux attachement à l'Écriture : certains auteurs comme Semler distinguent même nettement le lien croyant du fidèle à la Bible (avec l'appel à une lumière intérieure surnaturelle) et l'cuvre scientifique du théologien. Il apparait là, note H.G. Reventlow, une sorte de docétisme, qui ne sera dépassé que par la réflexion herméneutique du $\mathrm{XIX}^{e}$ siècle (p. 63).

Cependant, dans la deuxième moitié du XviIr siècle, un tournant est pris : il n'est plus question de rejeter l'historique dans les ténèbres du non-sensé, de l'irrationnel. Michaëlis, dans la ligne de Montesquieu, donne une explication réfléchie des singularités du droit hébreu : il le montre comme raisonnable, eu égard aux circonstances de temps et de lieu. Ainsi pointe le concept de "progrès" moral et religieux; l'histoire devient une genèse du sens, un devenir de la raison (communication de A. R. Lömenbrück : « Johann David Michaëlis' Verdienst um die philologisch-historische Bibelkritik "). Hamann, en critiquant le concept kantien de "raison pure ", affirme de son côté que la raison est toujours dans l'histoire. Aussi, soutenir qu'il y a une pure religion de la raison lui parait relever de la même erreur que de dire qu'il existe une "langue naturelle ", en soi, hors des langues historiques (communication de O. Bayer : " Vernunftautorität und Bibelkritik in der Kontroverse zwischen Johann Georg Hamann und Immanuel Kant "). Quant à Herder, on sait qu'en réhabilitant la singularité de chaque tradition culturelle, il contribue également à poser avec acuité le problème des rapports entre le singulier de l'histoire et l'universel de la raison (communications de $M$. Bunge et de D. Gützen sur Herder). L'ensemble des communications aide 
donc le lecteur à comprendre, à travers les transformations de l'exégèse, l'élévation de l'histoire à la dignité d'objet philosophique, par l'inclusion dans le rationnel de ce qui en fut d'abord exclu : la contingence de l'événement historique.

2. L'axe spatial

Il existe dès le $\mathrm{xvr}^{e}$ siècle une "Europe des humanistes ", dont le réseau des correspondants d'Érasme, tracé naguère par Robert Mandrou, offre une première figure (Des humanistes aux hommes de science. XVI et XVIf siècles, Paris, 1973, cartes, p. 245). Ensuite, va naitre la République des Lettres qui, dès la fin du $\mathrm{XVI}^{\mathrm{e}}$ siècle, échange à travers l'Europe les informations érudites. Le recul du latin, un siècle après, ne tarit pas la circulation européenne des travaux les plus renommés, qui sont présentés dans des périodiques savants et connaissent souvent des traductions en diverses langues. Ainsi l'Allemagne reçoit-elle des impulsions venues de la critique de Richard Simon, de la critique textuelle et de l'apologétique anglaises, tandis que les Pays-Bas restent activement fidèles à leur tradition philologique; que Bâle, Genève, Zurich, naguère citadelles de l'orthodoxie, jouent un rôle important dans les échanges intellectuels de l'Europe (communications de J. Woodbridge sur la réception de R. Simon en Allemagne et de $O$. Menk sur Turrettini et Wettstein). Cette configuration n'amène qu'à mieux reconnaitre les spécificités des Lumières allemandes. Leur penchant rationaliste s'explique par le prestige des philosophies de Leibniz et de Wolf: le concept selon lequel le monde est le meilleur des mondes possibles ne prépare guère à accueillir la dramatique chrétienne du péché et du salut (voir la communication de $M$. Schloemann sur Baumgarten). Mais l'Allemagne piétiste n'a pas oublié la distinction luthérienne entre la Parole de Dieu, comme message de salut, et l'Écriture, trace humaine de cette Révélation. Cette conviction fondamentale maintient l'attachement cordial à la Bible, contre vents et marées rationalistes, et prépare la théologie libérale de l'expérience religieuse (voir la communication de G. Hornig sur Semler).

Malgré les grands mérites de ce volume, l'on regrettera l'absence d'information sur les rapports entre l'évolution de l'exégèse et l'essor des sciences physiques et naturelles au XVIII ${ }^{e}$ siècle. H.G. Reventlow écarte un peu trop vite, à mon avis, le point de vue de Klaus Scholder selon lequel le rationalisme scientifique a eu une grande influence sur l'évolution de l'exégèse (p. 58). Il était possible, au minimum, de rappeler l'impact de la réception du système copernicien sur l'herméneutique de la cosmologie biblique. II me semble aussi que ne sont pas suffisamment abordées des questions comme l'histoire des sciences du langage et du droit, l'évolution de la société allemande au XviII siècle. Des aperçus, même brefs, sur ces points contribueraient à insérer l'histoire de l'exégèse dans les mutations culturelles et les conflits socio-politiques de son temps, hors desquels elle perd de sa saveur d'époque, pour devenir l'inventaire de modèles herméneutiques, constituant au sein du passé des jalons pour la science future. L'éditeur des actes du colloque, H.G. Reventlow, a d'ailleurs donné un remarquable échantillon de la méthode suggérée ici, si l'on peut appeler échantillon l'imposant volume de 770 pages qu'il a consacré à examiner les rapports entre la politique et l'exégèse 
biblique en Angleterre (Bibelautorität und Geist der Moderne, Göttingen, 1980; trad. angl., The Authority of the Bible and the Rise of the Modern World, Philadelphie, Fortress Press, 1985). Ici, l'insertion souhaitée de l'exégèse dans l'histoire est effectuée dans plusieurs cas : à propos de l'unionisme de Turrettini, des travaux historiques de Semler, de l'intérêt de Michaëlis pour la philosophie du droit. Tel qu'il se présente, le volume éditant les actes du colloque de Wolfenbüttel constitue un grand pas en avant par rapport aux histoires de l'exégèse déjà existantes (Kraus, Kümmel). Il éclaire remarquablement le processus de la sécularisation occidentale dans son application à la Bible comme dans ses origines religieuses et il faut lui souhaiter la plus large diffusion.

François LAPLANCHE.

Bernard LEWIS, Le Langage politique de l'islam. Trad. de l'anglais par Odette GurtaRD. Paris, Gallimard, 1988. $14 \times 22,5,243$ p., index.

Ce livre est issu d'une série de conférences prononcées à l'Université de Chicago en 1986.

Si l'on veut comprendre quoi que ce soit à la politique de l'islam, aux révolutions et aux bouleversements perçus et exprimés en termes islamiques, il faut essayer d'abord de connaître la langue du discours politique des musulmans, la façon dont les mots sont employés et compris, les références dont ils sont chargés, le système des métaphores et allusions qui font partie intégrante de toute communication.

Sir Bernard Lewis commence par souligner l'importance unique de la communication verbale en islam car bien des choses qui, dans d'autres religions, s'expriment par l'art et la musique, s'expriment ici par la parole.

Bernard Lewis, tout au long de son étude, recense les differentes strates linguistiques qui ont influencé le vocabulaire politique islamique. Sur la base du Coran, des Traditions du Prophète (hadith), qui plongent elles-mêmes leurs racines dans l'Arabie ancienne et les croyances juives, chrétiennes et païennes qui y préexistaient à l'avènement de l'islam, sont venus se greffer d'autres apports du fait de l'extension de l'aire islamique (manuels persans et traités grecs puis apports des langues de la steppe $d u x^{e}$ au XIv siècle). Enfin, l'influence de l'Europe aux $x \mid X^{e}$ et $x x^{e}$ siècles fut relayée par les Ottomans. Premier groupe dirigeant musulman à affronter les faits et à lire la littérature politique moderne et donc à èprouver le besoin d'un nouveau vocabulaire pour à la fois discuter et administrer, les turcs instruits forgèrent des néologismes, en puisant dans les abondantes richesses lexicales de l'arabe classique, comme les Occidentaux puisaient dans le grec et le latin.

Bernard Lewis commence par quelques précieux rappels : 
- Dans toutes les langues, qui tirent leur vocabulaire intellectuel et politique de l'arabe classique, il n'y a pas de couples de mots correspondant au spirituel et au temporel, au laïc et à l'ecclésiastique, au religieux et au séculier (hormis les greffons occidentaux inventés au XIX siècle par les chrétiens libanais). Aujourd'hui, la notion même d'une juridiction séculière, hors de portée de la loi religieuse, est jugée comme la trahison suprême de l'islam.

- L'islam est toujours le critère suprême de l'identité et du loyalisme de groupe au-delà des définitions nationales contemporaines.

- L'islam demeure la base la plus acceptable de l'autorité, voire la seule base légitime.

- La seule histoire importante et valable est celle de la vie du Prophète et de ses successeurs immédiats. C'est le tréfonds de la conscience historique des musulmans où qu'ils soient. C'est l'âge d'or, le modèle, qui doit être imité.

Dans le premier chapitre intitulé "Métaphore et allusion", Bernard Lewis répertorie les métaphores les plus usuelles. En matière de pouvoir, si l'Occident utilise les métaphores spatiales verticales de haut et de bas, l'islam préfère les métaphores horizontales de centre et de périphérie. Il s'agit d'une société qui, en principe, rejette la hiérarchie et le privilège et où le pouvoir dépend du degré d'intimité avec le souverain. Les thèmes de naissance, de mort et de résurrection sont écartés à cause de leur connotation chrétienne, au profit des métaphores de la maladie et de la faiblesse. Aucune valeur n'est accordée à la jeunesse et tout le respect va aux vieillards. Les métaphores tirées de la famille, de la maisonnée, de la demeure ont une place prééminente. L'image du souverain père du peuple est absente à cause de la proximité du concept chrétien de Dieu le Père, chose impensable pour le musulman. Esclave est la métaphore courante pour décrire la relation de l'homme à Dieu.

Dans le deuxième chapitre, « Le corps politique », Bernard Lewis explique la signification musulmane de l'autorité politique.

Les conditions de naissance de l'analyse politique au Moyen Âge en Occident et en Orient sont diamétralement opposées. La civilisation chrétienne occidentale est née dans le chaos des invasions barbares dans un contexte politique dominé par deux événements opposés et inséparables, la chute de l'État romain et l'essor de l'Église chrétienne. Pour saint Augustin, premier théoricien politique chrétien, le corps politique était l'œuvre de l'homme donc mauvais et le gouvernement une punition du péché originel. Après tout, Caïn avait fondé la première cité. Dans cette conception tirée des anciens prophètes hébreux et modelée par les événements de l'époque, la défaite et la destruction étaient des instruments divins destinés à amener l'humanité à l'Église, qui seule pourrait la conduire au salut. Ce n'est qu'au XII' siècle que saint Thomas d'Aquin, reconnaissant l'émergence d'un système politique chrétien, accorde une valeur positive aux institutions politiques.

Inversement en islam, l'analyse politique ne commence pas avec la défaite et la chute mais avec le triomphe et l'essor d'un empire. Au lieu de l'éprouver, Dieu aide le musulman à remporter la victoire et à étendre sa foi au monde entier. L'autorité politique qui conduit le musulman à la victoire est donc un bien divin. Sont ainsi étudiés les termes qui désignent l'autorité et la communauté.

Dans le troisième chapitre, "Gouvernants et gouvernés", ce sont toutes les 
appellations des souverains et des sujets qui sont examinées. En principe, l'islam est une religion égalitaire qui ne reconnaît ni castes ni aristocraties (hormis les descendants du Prophète - et ils furent nombreux dès l'origine - qui sont exemptés de la plupart des impôts, sans aucun autre privilège). Il n'y eut jamais de castes et les aristocraties furent précaires et éphémères. Si le privilège n'est pas admis, une triple inégalité est imposée et réglementée :

- l'inégalité de statut du maitre et de l'esclave;

- l'inégalité de statut de l'homme et de la femme;

- l'inégalité de statut du musulman et du non-musulman.

Dans le quatrième chapitre, "La guerre et la paix », B. Lewis développe une conception majeure de l'islam. Le monde est partagé en deux : La Maison de l'islam, où s'imposent la domination et la loi de l'islam, et la Maison de la guerre qui couvre le reste du monde. Entre les deux existe un état de guerre moralement nécessaire, juridiquement et religieusement obligatoire jusqu'au triomphe final et inévitable de l'islam sur l'incroyance. Le djihad (effort), traduit habituellement par guerre sainte, est l'un des éléments de la profession de foi, une obligation imposée par Dieu à tous les musulmans.

Dans la langue diplomatique musulmane, salām, la paix, est toujours employée pour s'adresser à un autre état musulman, même en temps de guerre; avec un souverain non musulman, même allié, pour parler de paix on utilise sulh, la trêve.

L'ennemi principal est l'incroyant (le non-mulsulman) insoumis, qui diffère du dhimmi, juif ou chrétien qui a accepté de se soumettre à la domination musulmane et qui paie une capitation marquant son infériorité de statut. La guerre la plus dure est celle qui est menée contre l'apostat. Celui-là n'a le choix qu'entre le retour à l'islam et la mort. Rare contre les individus l'accusation fut fréquente contre les états. Les Mongols, superficiellement islamisés, continuèrent, à la tête d'états musulmans, à gouverner selon la loi mongole et à ignorer la loi islamique. C'est le pire des désastres, puisque, sous le semblant de l'islam, c'est la loi et la foi des musulmans qui sont détruites. Aux $x^{e} x^{e}$ et $x^{e}$ siècles, sous l'effet de la colonisation européenne, de son influence et de la modernisation législative, le même problème de la non-application de la loi islamique se posa et est encore posé. C'est donc une guerre sainte intérieure, un djihad révolutionnaire, qui peut se développer dans un pays islamique. Le président égyptien Sadate fut assassiné comme apostat en 1981 .

Dans le dernier chapitre, Bernard Lewis définit les limites de l'obéissance. Le sujet doit obéir à son souverain légitime, c'est une obligation religieuse définie et imposée par la Loi sainte. La désobéissance est un péché et un crime. Mais le souverain est lui aussi soumis à la loi et s'il exerce une domination illégitime ou injuste, il peut être déchu de son droit à l'obéissance. Ces deux questions brûlantes de la légitimité et de la justice, très discutées tout au long de l'histoire de l'islam, ont reçu des réponses variables, mais en termes modernes l'efficacité de la Loi sainte en tant que limitation du pouvoir du souverain, présente deux failles importantes :

- la Loi elle-même donne des pouvoirs autocratiques considérables;

- il n'y a pas de mécanisme pour appliquer les limitations.

Il en résulte que les deux traditions autoritaire et quiétiste, d'une part, activiste 
et révolutionnaire de l'autre, ont des racines tout aussi anciennes s'inspirant des différentes périodes de la carrière de Mahomet.

Sir Bernard Lewis conclut sur l'incapacité des concepts politiques occidentaux à apporter une solution aux maux des peuples islamiques. Les remèdes empruntés à l'Europe de l'Est et de l'Ouest, à l'Amérique du Nord et du Sud n'ont apporté que déceptions et interrogations. Aussi un nombre croissant de musulmans se tournent-ils vers ce qu'ils perçoivent comme étant leur propre passé, afin d'y trouver les clefs de leur futur bien-être.

Nicolas JENSE.

\section{EXPRESSIONS DU POLITQUE}

Andrew W. LEWIS, Le Sang royal: la famille capétienne et l'État France, $X^{e}$-XIV siècle. Trad. de l'anglais Jeannie CARLIER, préf. de Georges DuBY. Paris, Gallimard, 1986. $14 \times 23,448$ p. ("Bibliothèque des histoires ").

Année de commémoration capétienne, 1987 a apporté une riche moisson de publications sur la monarchie et le pouvoir royal dans la France médiévale. Mais l'ouvrage de A. W. Lewis n'est en rien une œuvre de circonstance. C'est un maître livre, en effet, qui est mis à la disposition des lecteurs français, le résultat de minutieuses recherches, appelé à faire date par la densité érudite de son propos, sur les notions de la dynastie et du pouvoir exprimées et vécues dans une période dominée par l'aristocratie et la mystique royale chrétienne. La « Bibliothèque des histoires ", en éditant ce livre après l'ouvrage de Colette Beaune, Naissance de la nation France, qui s'intéressait surtout à la conception de la nation et de la monarchie au bas Moyen Âge, nous offre un diptyque chronologique des idées du pouvoir dans le royaume de France. On nous permettra de dire ici notre préférence pour la rigueur et l'érudition de l'étude d'A. Lewis, qui est animé par la volonté de poursuivre une enquête constamment démonstrative et vérificatrice.

L'érudition exemplaire d'A. Lewis est sans complaisance, au détriment parfois de la compréhension immédiate du propos de l'auteur. L'analyse progresse pas à pas, et n'aborde jamais un nouveau développement avant d'avoir établi la liste des objections possibles à l'étape précédente de la démonstration. Ce souci est 